\title{
Dietary Bacillus subtilis supplementation alleviates alcohol-induced liver injury by maintaining intestinal integrity and gut microbiota homeostasis in mice
}

\author{
MEIQI ZHAO ${ }^{1}$, CHUANAI CHEN $^{2}$, ZHOUJIE YUAN ${ }^{1,3}$, WENWEN LI $^{1,3}$, MOHAN ZHANG $^{1}$, \\ NAILING CUI ${ }^{1}$, YITAO DUAN ${ }^{2}$, XIAOQIAN ZHANG ${ }^{1,3}$ and PENG ZHANG ${ }^{2}$ \\ ${ }^{1}$ School of Clinical Medicine, Weifang Medical University, Weifang, Shandong 261031 ; ${ }^{2}$ School \\ of Medicine, Nankai University, Tianjin 300071; ${ }^{3}$ Department of Gastroenterology, Affiliated \\ Hospital of Weifang Medical University, Weifang, Shandong 261031, P.R. China
}

Received August 20, 2020; Accepted March 24, 2021

DOI: $10.3892 /$ etm.2021.10747

\begin{abstract}
Alcoholic liver disease (ALD) is a worldwide health problem with limited therapeutic options, which is associated with gut-derived endotoxins, particularly lipopolysaccharide (LPS) and intestinal microbiota dysbiosis. Recently, probiotics, synbiotics and other food additive interventions have been shown to be effective in decreasing or preventing the progression of ALD. Bacillus subtilis (B. subtilis) and its metabolic products are widely used as food additives to maintain intestinal health, but the protective effects of B. subtilis against alcohol-induced liver injury are poorly understood. In the present study a chronic alcohol-induced liver injury model was constructed based on the Lieber-DeCarli diet and it aimed to determine whether dietary B. subtilis supplementation may alleviate alcohol-induced liver injury. Results revealed that prophylactic $B$. subtilis supplementation partially restored gut microbiota homeostasis and relieved alcohol-induced intestinal barrier injury, which significantly decreased the translocation of bacterial endotoxins to the blood. In addition, the decreased serum LPS alleviated hepatic inflammation via the toll-like receptor 4 pathway, resulting in improved hepatic structure and function. These results demonstrated that dietary B. subtilis supplementation imparts novel hepatoprotective functions by improving intestinal permeability and homeostasis.
\end{abstract}

Correspondence to: Professor Xiaoqian Zhang, Department of Gastroenterology, Affiliated Hospital of Weifang Medical University, 2428 Yuhe Road, Weifang, Shandong 261031, P.R. China E-mail: nku311@126.com

Dr Peng Zhang, School of Medicine, Nankai University, 94 Weijin Road, Tianjin 300071, P.R. China

E-mail: zpeng@mail.nankai.edu.cn

Key words: Bacillus subtilis, alcohol-induced liver injury, intestinal integrity and homeostasis, gut microbiota

\section{Introduction}

According to World Health Organization estimates, alcohol consumption resulted in 3 million alcohol-attributable deaths globally (5.3\% of all deaths worldwide in 2016) and $5.1 \%$ of the global disease burden in 2016 (1). Alcohol abuse represents the most common inducer of liver disease and is a considerable contributor to the global burden of disease (2). More seriously, harmful alcohol consumption is well recognized to be the major cause of alcoholic liver disease (ALD) and liver cirrhosis worldwide (3). A previous study confirmed that drinking pattern, alcohol quantity and nutritional status directly influence the progression of alcoholic liver disease (4). In addition, it is intriguing to note that intestinal homeostasis has been recently shown to serve a pivotal role in modulating the development of alcohol-induced liver injury (5-7).

Alcohol consumption may cause an imbalance in intestinal flora by notable altering bacterial abundance and diversity (8), and the profiles of disordered gut microbiota involved in alcohol-related liver disease have been well characterized with an increase in Proteobacteria and Fusobacteria and a decrease in Bacteroidetes and Lactobacillus species $(9,10)$. Another predominant feature of ALD pathogenesis is leakiness of the gut barrier, resulting in the translocation of enterobacteria and bacterial products (particularly endotoxemia,LPS) from the intestinal lumen into the liver (11). These pathogen-associated molecular patterns (PAMPs; e.g., LPS) potently activate toll-like receptors (TLRs, particularly TLR4) and lead to nuclear factor- $\kappa \mathrm{B}$ $(\mathrm{NF}-\kappa \mathrm{B})$ and inflammatory cytokine production $(8,12,13)$. These cytokines consequently attract leukocytes in liver tissues, which further promotes hepatic injury $(14,15)$. Therefore, it can be reasonably inferred that improving alcohol-induced intestinal dysfunction may represent a key for effective therapy.

Notably, liver steatosis is the common response to excessive alcohol exposure and is characterized by the deposition of fat and abnormal lipid metabolism in hepatocytes $(16,17)$, of which fatty-acid transport and oxidation are disturbed. Alcohol enhances upregulation of hepatic fatty acid transporters (18), including CD36/fatty acid translocase and fatty acid transport protein. In addition, previous studies have revealed that alcohol 
or its metabolic products inactivate the peroxisome proliferator activated receptor alpha (PPAR- $\alpha$ ) and stimulate the peroxisome proliferator activated receptor gamma (PPAR- $\gamma$ ), which acts directly with the retinoid $\mathrm{X}$ receptor and participates in regulating fatty-acid transport and oxidation $(19,20)$. Meanwhile, the inflammation responses stimulated by gut-derived LPS exaggerate the alcohol-induced liver function disorders (21), likely worsen the lipid metabolism disturbance in the liver.

Previous studies have discovered an efficient nutraceutical intervention via immunomodulatory probiotics and prebiotics to prevent gut barrier disruption and modulate intestinal microbiome balance (22-25). To date, Bacillus subtilis (B. subtilis) and its metabolic products, including nattokinase, carbohydrase and protease, are widely used as food ingredients and food additives. $B$. subtilis-based probiotics are increasingly being used to maintain intestinal health, and the beneficial attributes of $B$. subtilis are credited to its ability to produce antimicrobial peptides and small extracellular effector molecules and to interact with a host with the aid of adhesion and attachment features (26-28). Additionally, several important clinical trials have demonstrated that B. subtilis-based probiotic supplementation may improve perturbed gut microbiota and even relieve the adverse effects of antibiotic-associated diarrhea $(29,30)$. In conclusion, dietary B. subtilis supplementation have great potential to restore disordered gut microbiota; however, the effects of dietary B. subtilis supplementation on alcohol-induced liver injury have been seldom reported. The present study characterized the efficacy of B. subtilis strains as potential beneficial probiotics in alcohol-induced liver injury and characterized the underlying mechanisms.

\section{Materials and methods}

Bacterial strains and cultures. The Bacillus strain used in the present study was B. subtilis (CMCC 1.3358), which was commercially available from China General Microbiological Culture Collection Center. For experimental purposes, single colonies of the Bacillus strain were grown in LB broth at $37^{\circ} \mathrm{C}$ overnight with shaking (200 rpm). Next, overnight cultures were diluted (1:100) in LB broth and left to grow at $37^{\circ} \mathrm{C}$ with shaking (200 rpm) until OD600 was reached 0.5-0.6 ( 2-3 h). Bacillus strains were then pelleted by centrifugation $(6,000 \mathrm{x} \mathrm{g})$ at room temperature for 2 min, diluted in Fresh PBS solution and mixed to obtain the appropriate bacterial density (B. subtilis: $\left.5 \times 10^{8} \mathrm{CFU} / \mathrm{ml}\right)$.

Animal study. The animal experiments in the present study were approved by the Ethics and Clinical Research Committee of Nankai University (Project IRM-DWLL-2016121). A total of 40 male C57BL/6J mice (20-22 g; 4-6 weeks old) used in the present study were purchased from the Beijing Hfk Bioscience Co., Ltd. Animals had a sanitary status of SPF and were housed in regulation cages $\left(22 \pm 1^{\circ} \mathrm{C}\right.$, relative humidity of $50 \pm 10 \%$ and 12-h light/dark cycle) with ad libitum access to food and water. All the mice were subdivided into groups (10 mice in each group): Group 1, Control (Ctrl); group 2, ethyl alcohol (EtOH) group; group 3, ethyl alcohol and B. subtilis supplementation (EtOH+BS), group 4, ethyl alcohol and PBS (EtOH+PBS). The alcohol-induced liver injury model was constructed in mice using the Lieber-DeCarli diet as previously described (31). In brief, all mice were allowed to acclimatize to the laboratory conditions for 2 days and to a liquid diet for 5 days, and two mice were housed in each cage. During the acclimatization to liquid diet, prophylactic supplementation with $B$. subtilis or PBS were performed daily. Following the acclimatization, the alcohol-fed mice were fed with a diet containing 3\% (vol/vol) ethanol for 10 days, while the $\mathrm{Ctrl}$ mice received an isocaloric amount of maltodextrin as pair-fed control. The mice in the $\mathrm{EtOH}+\mathrm{BS}$ group were administered bacterial suspension in PBS $(200 \mu \mathrm{l})$ by gavage every day. Body weights were measured every other day. On day 16 , the mice received a single dose of ethanol via oral gavage ( $5 \mathrm{~g} / \mathrm{kg}$ body weight), followed by a $9 \mathrm{~h}$-fast, and were sacrificed by anesthesia with isoflurane (4\%) prior to excision of tissue samples.

Intestinal permeability assays. To determine the intestinal permeability, fluorescein isothiocyanate (FITC)-dextran (4 kDa; Sigma-Aldrich; Merck KGaA) was orally administered (600 mg/kg body weight) to mice $4 \mathrm{~h}$ before sacrifice. Blood samples were collected quickly and subsequently centrifuged $\left(1,500 \mathrm{x} \mathrm{g}, 4^{\circ} \mathrm{C}, 15 \mathrm{~min}\right)$ to prepare serum. Fluorescence was recorded using a spectrophotometer (Tecan Group, Ltd.) at an excitation wavelength of $485 \mathrm{~nm}$ and emission wavelength of $528 \mathrm{~nm}$.

Biochemical analysis. Serum aspartate transaminase (AST) and alanine transaminase (ALT) were measured using the Infinity reagent (cat. nos. TR71121 and TR70121; Thermo Fisher Scientific). Hepatic triglyceride (TG) levels were measured using the Triglyceride Liquid Reagents kit (cat. no. BC0625, Beijing Solarbio Science \& Technology Co., Ltd.), according to the manufacturer's protocols. Hepatic lipid peroxidation was quantified by measuring malondialdehyde (MDA) using Micro MDA assay kit (cat. no. BC0025, Beijing Solarbio Science \& Technology Co., Ltd.), and the oxidative stress of liver tissues was characterized by testing the decreased glutathione content using a reduced glutathione (GSH) assay kit (cat. no. BC1175, Beijing Solarbio Science \& Technology Co., Ltd.). Serum LPS was determined using the Mouse Lipopolysaccharides (LPS) ELISA kit (cat. no. CSB-E13066m, Cusabio). The fecal IgA levels were quantified using a Mouse sIgA ELISA kit (cat. no. CSB-E08413m, Cusabio). The expression level of IL-6 in serum was determined using a Mouse IL-6 ELISA kit (cat. no. SEKM-0007, Beijing Solarbio Science \& Technology Co., Ltd.). Serum levels of total cholesterol (TCH), low-density lipoprotein cholesterol (LDL-C), and high-density lipoprotein cholesterol (HDL-C) were detected colorimetrically by mindray $2000 \mathrm{M}$ autoanalyzer according to the manufacturer's protocols.

Reverse transcription-quantitative polymerase chain reaction $(R T-q P C R)$. Total liver and colon RNA were extracted using TRIzol reagent (Invitrogen; Thermo Fisher Scientific, Inc.), and the total RNA concentration was quantified using the NanoPhotometer N50 (Implen NanoPhotometers), and cDNA was synthesized at $42^{\circ} \mathrm{C}$ for $2 \mathrm{~min}$ on an Mastercycler nexus PCR using PrimeScript RT reagent kit with gDNA Eraser (Takara Bio, Inc.). The synthesized cDNA was stored at $-20^{\circ} \mathrm{C}$, and RT-qPCR was conducted on a LightCycler ${ }^{\circledR} 96$ system (Roche Diagnostics) using a TB Green Premix Ex Taq II (Tli RNaseH 
Table I. Primers for reverse transcription-quantitative polymerase chain reaction.

\begin{tabular}{|c|c|c|}
\hline Genes & Forward primers & Reverse primers \\
\hline$I L-6$ & CCACTTCACAAGTCGGAGGCTTA & CCAGTTTGGTAGCATCCATCATTTC \\
\hline$M C P-1$ & CCAGCCTACTCATTGGGATCA & CTTCTGGGCCTGCTGTTCA \\
\hline$T N F-\alpha$ & ACССТCACACTCAGATCATCTTC & TGGTGGTTTGCTACGACGT \\
\hline TLR4 & GGCATGGCATGGCTTAAACC & CATCGGTTGATCTTGGGAGAATT \\
\hline$N F-\kappa B$ & GGGACTATGACTTGAATGCG & ATACGCTGACCCTAGCCTG \\
\hline Zo-1 & GACCTTGATTTGCATGACGA & AGGACCGTGTAATGGCAGAC \\
\hline Occludin & GAAAGTCCACCTCCTTACAGA & CGGATAAAAAGAGTACGCTGG \\
\hline Muc2 & GCTCGGAACTCCAGAAAGAAG & GCCAGGGAATCGGTAGACAT \\
\hline $\operatorname{Reg} 3 b$ & GGAATTCGATGTCCAAAA & CGGTCGACGTGAACTTTGCAGACATA \\
\hline $\operatorname{Reg} 3 g$ & TTCCTGTCCTCCATGATCAAA & CATCCACCTCTGTTGGGTTC \\
\hline $18 s$ & AGGGGAGAGCGGGTAAGAGA & GGACAGGACTAGGCGGAACA \\
\hline
\end{tabular}

$M C P-1$, monocyte chemoattractant protein- $1 ; T N F-\alpha$, tumor necrosis factor $\alpha ; T L R 4$, toll-like receptor $4 ; N F-\kappa B$, nuclear factor- $\kappa \mathrm{B}$.

Plus; Takara Bio, Inc.). The thermocycling conditions were as follows: Initial denaturation for $10 \mathrm{~min}$ at $95^{\circ} \mathrm{C}$, then 40 cycles of $10 \mathrm{sec}$ at $95^{\circ} \mathrm{C}, 10 \mathrm{sec}$ at $62^{\circ} \mathrm{C}$ and $10 \mathrm{sec}$ at $72^{\circ} \mathrm{C}$, followed by $95^{\circ} \mathrm{C}$ for $60 \mathrm{sec}$ and dissociation curve analysis. The primer sequences are listed in Table I. Relative gene expression was normalized to the $18 \mathrm{~S}$ and calculated by the $2^{-\Delta \Delta \mathrm{Ct}}$ method (32).

Western blotting. Total protein was extracted from livers using a RIPA buffer containing $1 \%$ protease inhibitor and $1 \%$ phenylmethylsulfonyl fluoride (cat. no. R0010; Beijing Solarbio Science \& Technology Co., Ltd.) and was measured by the BCA assay. Total protein (50 $\mu \mathrm{g}$ per lane) was separated by $10 \%$ SDS-polyacrylamide gel, transferred onto a polyvinylidene difluoride membrane (Whatman plc), and blocked with $5 \%$ skimmed milk at room temperature for $1 \mathrm{~h}$. Membranes were immunostained with primary antibodies against TLR4 (cat. no. 14358; dilution, 1:1,000; CST) at $4^{\circ} \mathrm{C}$ overnight, as well as antibody against reference protein $\beta$-actin (cat. no. K200058M; dilution, 1:1,000; Beijing Solarbio Science \& Technology Co., Ltd.). Following incubation with a goat anti-rabbit horseradish peroxidase-conjugated secondary antibody (cat. no. SE134; dilution, 1:5,000; Beijing Solarbio Science $\&$ Technology Co., Ltd.) was carried out at room temperature for $1 \mathrm{~h}$. Immunoreactive proteins were stained with ECL Western Blotting Substrate (Beijing Solarbio Science \& Technology Co., Ltd.). Images were digitalized using the ChemiDoc ${ }^{\mathrm{TM}}$ XRS system (Bio-Rad Laboratories, Inc.). $\beta$-actin (Beijing Solarbio Science \& Technology Co., Ltd.) was used as an internal standard.

Histopathological observation. For the histological analysis, the liver and colonic tissues were stained with hematoxylin and eosin (H\&E). In brief, tissues were fixed in $10 \%$ formalin at room temperature for $24 \mathrm{~h}$, and paraffin-embedded sections $(5 \mu \mathrm{m})$ were stained with hematoxylin $(10 \mathrm{~min})$ and eosin $(30 \mathrm{sec})$ at room temperature.

DNA extraction and 16S rRNA amplification sequencing. Total genomic DNA was extracted from 150-200 mg of stool samples using the QIAamp PowerFecal DNA kit (Qiagen GmbH). The hypervariable V3-V4 region (341F and 805R) of the the prokaryotic 16S rRNA gene was amplified. Following purification, these amplicons were equally combined and then subjected to a sequencing library preparation according to the manufacturer's protocols. DNA concentration and quality were determined by the NanoPhotometer N50 (Implen NanoPhotometers) and Qubit Fluorometer (Thermo Fisher Scientific, Inc.). Deep DNA pyrosequencing procedures were performed on a paired-end Illumina MiSeq PE300 (2x300 bp) platform at the Novogene Corporation, according to the manufacturer's protocols. These raw sequences were processed following the QIIME (v1.9.1) pipeline (33), and the analysis of gut microbiota diversity and composition of fecal samples was determined, of which Shannon indexes and Simpson indexes were calculated with the operational taxonomic unit (OTU) table (34). The $\beta$ diversity of fecal microbiota was assessed by principal coordinate analysis based on Bray-Curtis distance.

Statistical analysis. All experimental results were obtained from at least three independent times, and representative data are presented as the mean \pm standard deviation. One-way analysis of variance was used to determine whether the groups were statistically different $(\mathrm{P}<0.05)$. GraphPad Prism 7.0 (GraphPad Software, Inc.) was applied to all statistical analysis.

\section{Results}

Dietary B. subtilis supplementation alleviates alcohol-induced injury. To investigate the effect of the prophylactic dietary B. subtilis supplementation on alcohol-induced injury, a murine model was constructed as shown in Fig. 1A. Notably, alcohol feeding caused continuous body weight loss in the EtOH group compared with the Ctrl group; however, dietary B. subtilis supplementation notably prevented alcohol-induced body weight loss (Fig. 1B). Following these mice being sacrificed, it was observed that ethanol feeding significantly increased the murine liver weight and the liver-body weight ratio compared with Ctrl mice, and dietary B. subtilis supplementation 


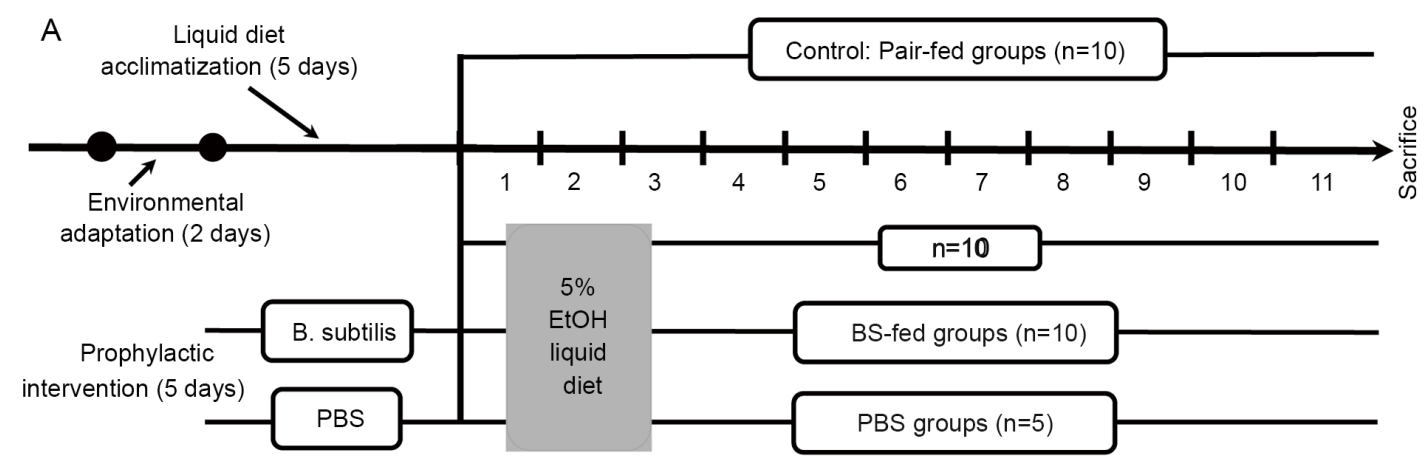

B

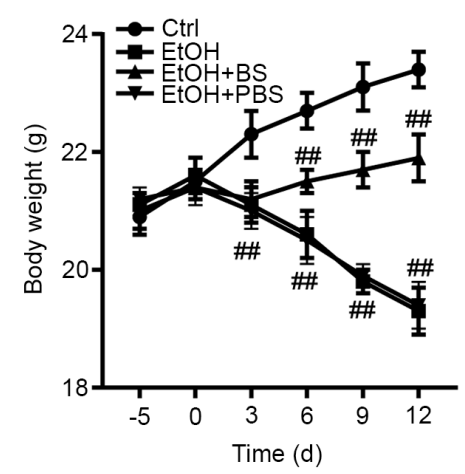

C

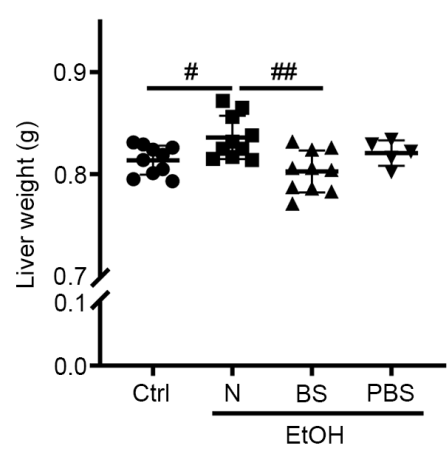

$\mathrm{D}$

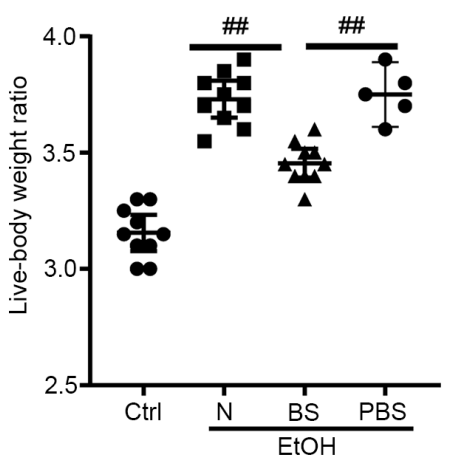

Figure 1. Effects of BS supplementation on alcohol-induced liver injury. (A) Animal feeding schedule: Mice were divided into four groups and administered $3 \% \mathrm{EtOH}, 3 \% \mathrm{EtOH}+\mathrm{BS}, 3 \% \mathrm{EtOH}+\mathrm{PBS}$ or pair-fed Ctrl. BS was administered by gavage (1x10 $10^{\circ} \mathrm{CFU} /$ mice). (B) Body weight. (C) Liver weight. (D) Liver-body weight ratio (\%). Data are presented as the mean \pm standard deviation of at least three independent experiments. ${ }^{\#} \mathrm{P}<0.05$; ${ }^{\# \#} \mathrm{P}<0.01$. EtOH, ethyl alcohol; BS, B. subtilis supplementation; PBS, phosphate-buffered saline; Ctrl, control.

significantly decreased the liver weight (Fig. 1B and C). Notably, it was found that supplementation with PBS-bacterial vehicle did not cause notable effects in EtOH-fed mice (Fig. 1B-D); therefore, the effects of PBS supplementation in EtOH-fed mice were excluded from further experiments. In conclusion, dietary B. subtilis supplementation likely alleviated alcohol-induced injury.

Dietary B. subtilis supplementation alleviates alcohol-induced liver injury. The development of alcohol-induced liver injury (EtOH group, Fig. 2A) was confirmed, as indicated by notably increased levels of ALT, AST and hepatic triglycerides compared with the Ctrl group (Fig. 2B-E). In addition, it was investigated whether dietary $B$. subtilis supplementation may relieve alcohol-induced liver injury in mice. Microscopic morphological examination confirmed the protective effect of dietary B. subtilis supplementation with a decrease in clear vacuoles and neutrophil infiltration in H\&E-stained images (Fig. 2A). Dietary B. subtilis supplementation significantly decreased hepatic triglyceride levels in treated mice compared with EtOH-fed mice (Fig. 2C). Additionally, the hepatic dysfunction induced by alcohol was improved as demonstrated by decreased serum ALT and AST levels (Fig. 2B and E), as well as the partial decrease in serum TCH and LDL-C level (Fig. S1). Furthermore, hepatic oxidative stress was induced by alcohol consumption with increased levels of hepatic thiobarbituric acid-reactive substances (TBARS) in the EtOH mice $(1.53 \pm 0.07 \mu \mathrm{mol} / \mathrm{g}$ liver) compared with the Ctrl mice $(1.03 \pm 0.09 \mu \mathrm{mol} / \mathrm{g}$ liver), and dietary $B$. subtilis supplementation significantly decreased hepatic MDA levels to $1.17 \pm 0.06 \mu \mathrm{mol} / \mathrm{g}$ liver (Fig. 2D). GSH depletion was another trait of ethanol-induced liver injury, and the administration of $B$. subtilis notably increased the content of reduced GSH (Fig. S2). Consistent with these protective effects, dietary B. subtilis supplementation significantly decreased the expression of peroxisome proliferator-activated receptor- $\gamma$ (PPAR- $\gamma$ ) and fatty acid translocase (CD36; Fig. 2F and G), which likely ameliorated the hepatic lipid dysfunction induced by alcohol.

Dietary B. subtilis supplementation preserves the intestinal barrier. Previous studies have characterized gut barrier dysfunction in alcohol-induced endotoxemia and liver damage (35-37), and disordered intestinal integrity may be due to the direct injury induced by alcohol and indirect inflammatory reactions $(38,39)$. In the present study, alcohol consumption resulted in a significant increase in intestinal permeability. Specifically, serum FITC concentrations increased to $1,183 \pm 76.18 \mathrm{ng} / \mathrm{ml}$ from $439.2 \pm 23.53 \mathrm{ng} / \mathrm{ml}$ in Ctrl mice (Fig. 3A), and evidence of colon damage was noted through microscopic morphological examination of H\&E-stained samples (Fig. 3B). However, dietary B. subtilis supplementation significantly restored intestinal permeability to a certain degree, decreasing FITC concentrations to $786.8 \pm 45.8 \mathrm{ng} / \mathrm{ml}$ (Fig. 3A). In addition, the damaged gut barrier resulted in the translocation of LPS from lumen to blood, and dietary B. subtilis supplementation significantly decreased LPS levels to $57.43 \pm 3.35 \mathrm{ng} / \mathrm{ml}$ from $88.04 \pm 7.6 \mathrm{ng} / \mathrm{ml}$ in $\mathrm{EtOH}$ mice (Fig. 3C). Consistent with amelioration of intestinal barrier dysfunction, dietary B. subtilis supplementation significantly restored Muc2, Zo-1 and occludin expression levels in the colon (Fig. 3D). Additionally, alcohol consumption 

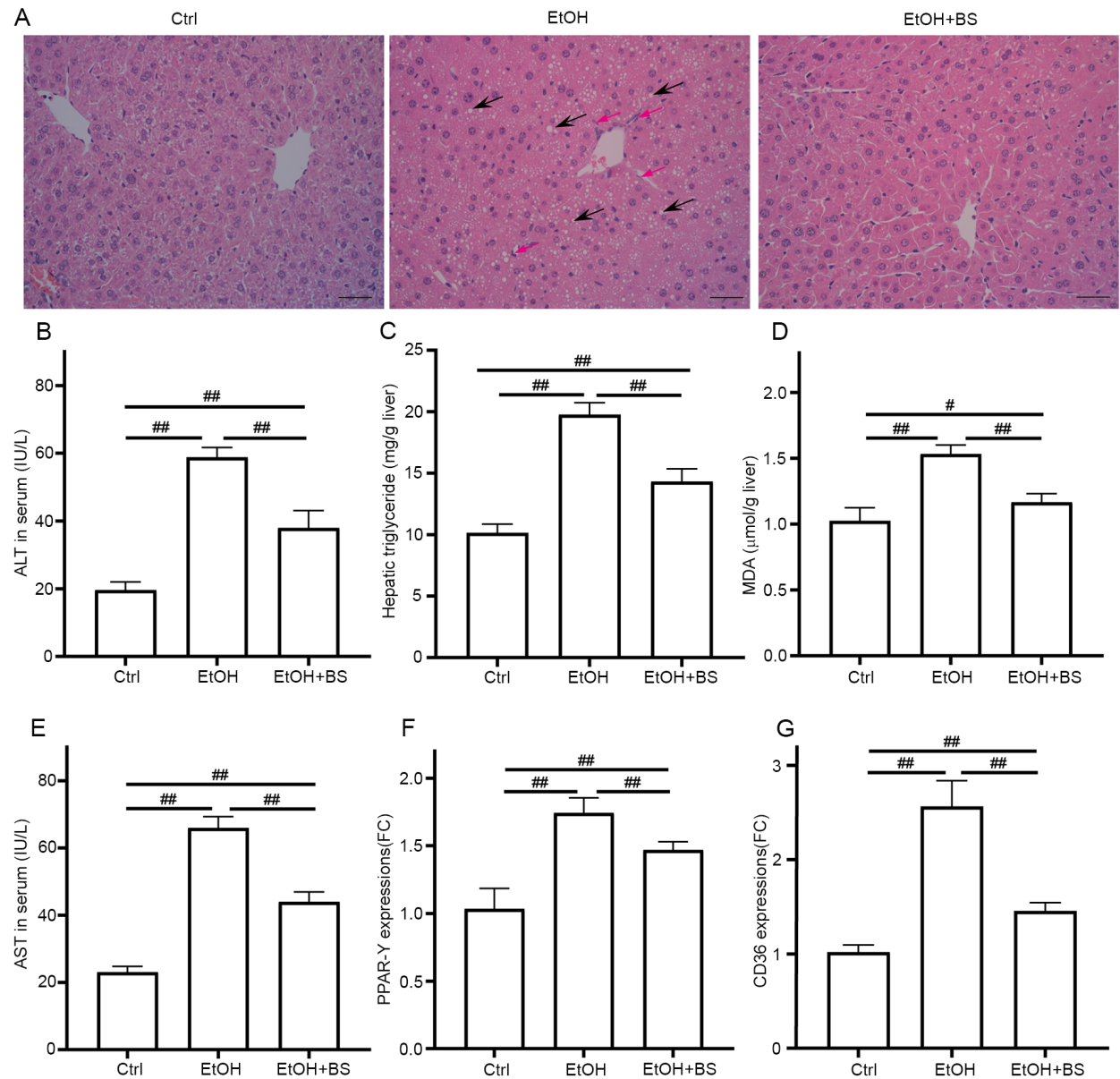

Figure 2. BS supplementation alleviates alcohol-induced liver injury. (A) Representative photomicrographs of hematoxylin and eosin-stained liver sections, black arrows show the steatosis and red arrows show neutrophil infiltration (magnification, x200). (B) Serum ALT levels. (C) Hepatic triglyceride levels. (D) Hepatic TBARS levels. (E) Serum AST levels. (F and G) Hepatic expression of gene PPAR- $\gamma$ and $C D 36$, respectively. Data are presented as the mean \pm standard deviation of at least three independent experiments. ${ }^{\# P} \mathrm{P}<0.05 ;{ }^{\# \#} \mathrm{P}<0.01$. BS, B. subtilis supplementation; ALT, alanine transaminase; TBARS, thiobarbituric acid-reactive substances; AST, aspartate transaminase; PPAR- $\gamma$, peroxisome proliferator activated receptor gamma; EtOH, ethyl alcohol; Ctrl, control; MDA, malondialdehyde.
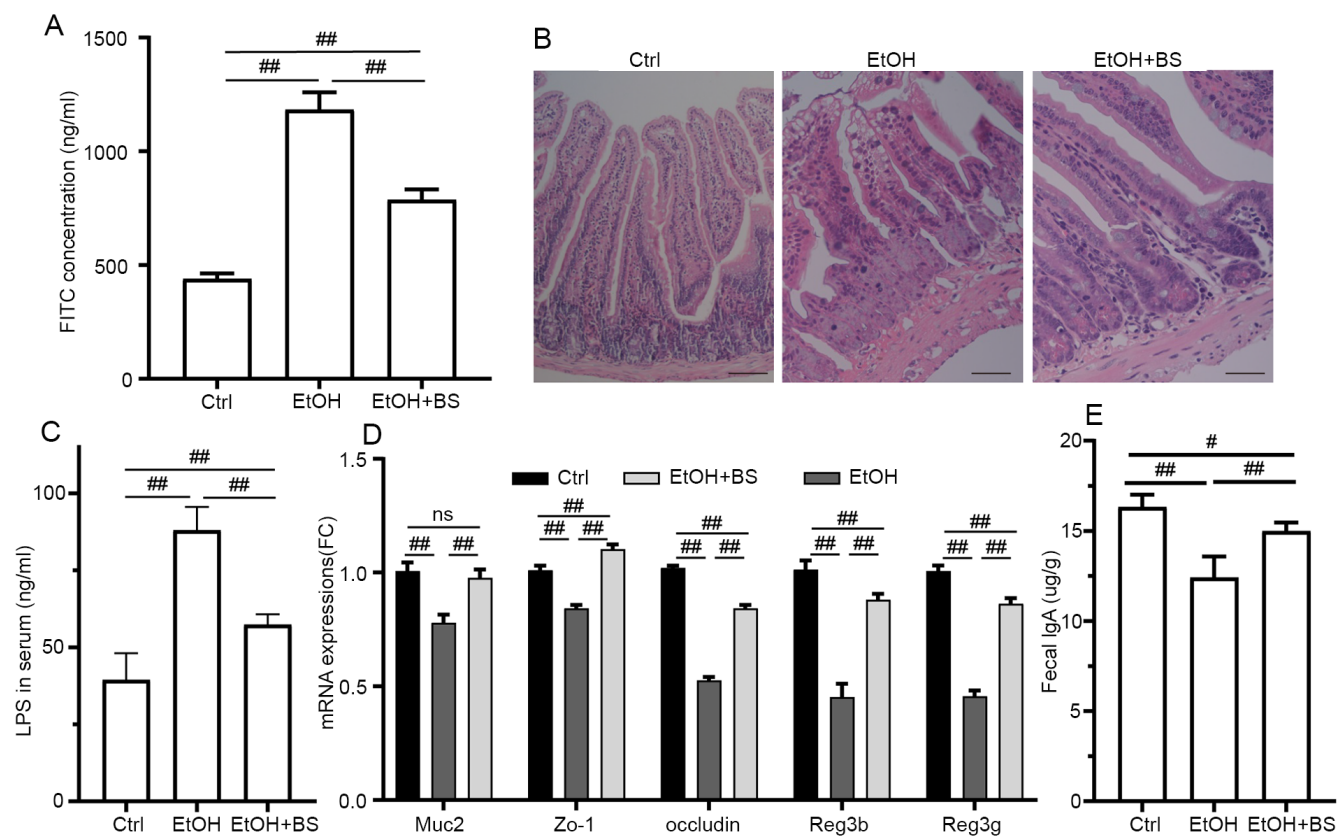

Figure 3. BS supplementation improves alcohol-induced colonic injury. (A) Plasma concentration of FITC-dextran (administered via oral gavage 4 h prior to euthanasia). (B) Representative photomicrographs of hematoxylin and eosin-stained colonic sections (magnification, x200). (C) Serum LPS levels. (D) Colonic expression of Muc2,Zo-1, occludin, Reg3b and Reg3g genes, respectively. (E) Fecal IgA levels. Data are presented as the mean \pm standard deviation of at least three independent experiments. ${ }^{~} \mathrm{P}<0.05$; ${ }^{\# \#} \mathrm{P}<0.01$. BS, B. subtilis supplementation; LPS, lipopolysaccharide; IgA, immunoglobulin A; EtOH, ethyl alcohol; Ctrl, control. 


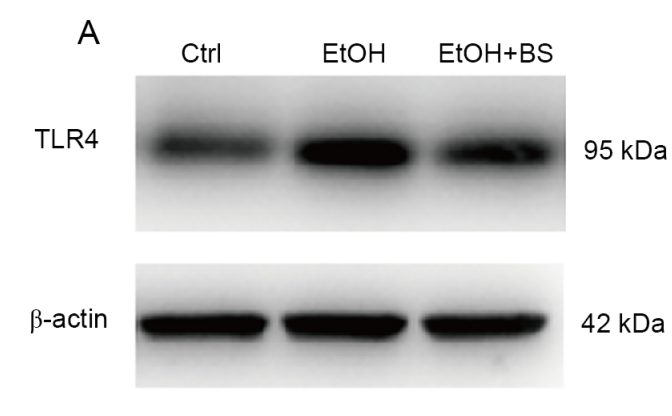

$$
\text { C }
$$

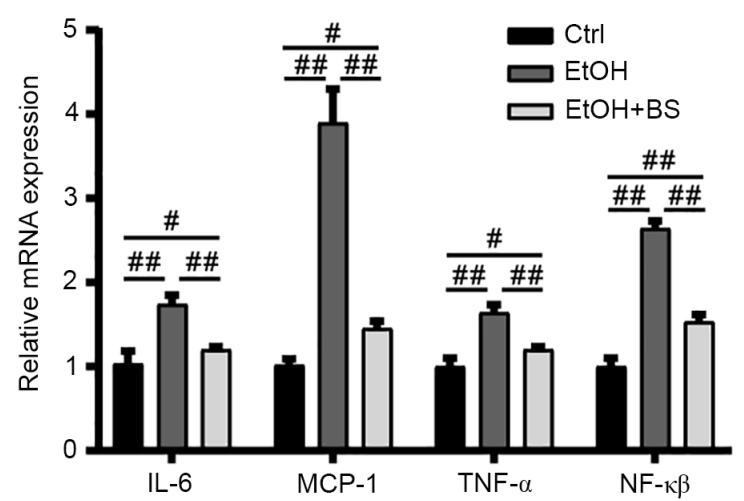

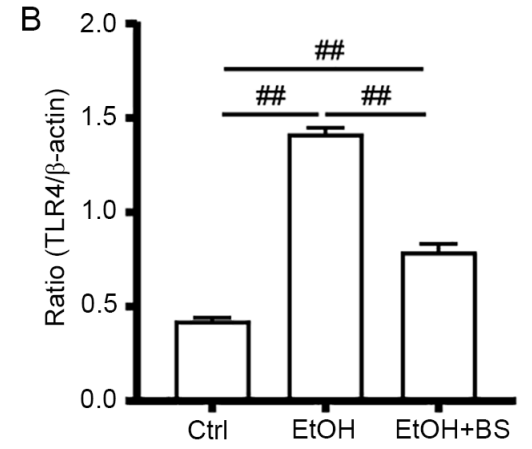

$\mathrm{D}$

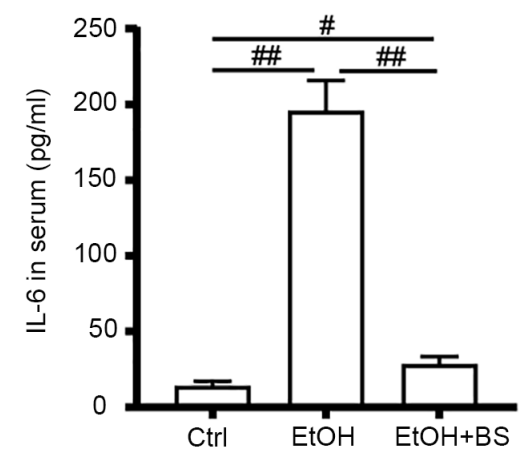

Figure 4. BS supplementation alleviates alcohol-induced liver inflammation. (A) Protein expression of TLR4. (B) Values of TLR4 protein expression were normalized to those of $\beta$-actin as the control. (C) Hepatic gene expression of proinflammatory cytokines $T N F-\alpha, N F-\kappa \beta$ and $I L-6$ and the chemokine $M C P$ - 1 . (D) Serum IL-6 levels. Data are presented as the mean \pm standard deviation of at least three independent experiments. ${ }^{\#} \mathrm{P}<0.05$; ${ }^{\# \#} \mathrm{P}<0.01$. BS, B. subtilis supplementation; TLR4, toll-like receptor 4; TNF- $\alpha$, tumor necrosis factor alpha; $N F-\kappa B$, nuclear factor- $\mathrm{B}$; $M C P-1$, monocyte chemoattractant protein-1; Ctrl, control; EtOH, ethyl alcohol.

notably damaged immunoglobulin A ( $\operatorname{Ig} \mathrm{A})$ production and secretion with fecal IgA levels decreased by $24 \%$ in the $\mathrm{EtOH}$ group compared with the Ctrl group, and dietary B. subtilis supplementation significantly restored the level of IgA secretion (Fig. 3E). Furthermore, dietary B. subtilis supplementation notably reversed the alcohol-induced decrease in Reg3g and Reg $3 b$ mRNA expression to levels equivalent to those noted in the Ctrl group (Fig. 3D), thereby improving intestinal barrier function.

Dietary B. subtilis supplementation ameliorates alcohol-induced liver inflammation via the TLR4 pathway. Alcohol consumption increases gut bacteria-derived PAMPs (e.g., LPS) in the bloodstream, and PAMPs trigger hepatic inflammation, which contributes toward the development of alcohol-induced liver diseases (21). In the present study, alcohol consumption increased LPS levels in the serum, which stimulated TLR4 to induce the release of critical proinflammatory cytokines that are required to activate potent immune responses. Alcohol-induced increases in TLR4 protein levels were accompanied by significant upregulation of the mRNA levels of TLR4 target genes (Fig. 4A-C), including $N F-\kappa B$, tumor necrosis factor alpha $(T N F-\alpha), I L-6$ and monocyte chemoattractant protein-1 (MCP-1), indicating activation of the alcohol-induced hepatic TLR4 pathway. However, dietary B. subtilis supplementation significantly decreased hepatic TLR4 protein levels by $53 \%(\mathrm{P}<0.01)$ with concomitant significant decreases in TLR4-regulated mRNA levels of $N F-\kappa B, T N F-\alpha, I L-6$ and $M C P-1(\mathrm{P}<0.01)$ in the treated group, compared with the EtOH group (Fig. 4C).
Dietary B. subtilis supplementation improves alcohol-induced gut microbial dysbiosis. It has been reported that dysbiosis of the gut microbiome and metabolome is associated with the progression of alcohol-induced liver injury $(5,40,41)$. The present study characterized the protective effects of dietary $B$. subtilis supplementation on alcohol-induced perturbation of intestinal microbiota composition. OTU-based principal coordinate analysis revealed that the gut microbiota structures of each tested group exhibited individual variations and that the experimental groups significantly differed from the Ctrl group (Fig. 5A). The diversity of gut microbiota in EtOH-fed mice compared with Ctrl mice was significantly decreased as evidenced by decreased Shannon indexes and increased Simpson indexes; however, dietary B. subtilis supplementation reversibly increased microbiota diversity (Fig. 5B). Analysis of the gut microbiota at phylum levels revealed a significantly altered composition of gut microbiota in Ctrl, EtOH and EtOH + BS groups (Fig. 5C). The phyla Firmicutes, Bacteroidetes and Proteobacteria dominated the fecal microbial communities (Fig. 5C), and alcohol consumption significantly increased the abundance of Proteobacteria and decreased that of Firmicutes (Fig. 5D-F); however, dietary B. subtilis supplementation markedly decreased the level of Proteobacteria to that noted in the Ctrl group (Fig. 5F). Notably, further analysis at the family level revealed a marked decrease in Lachnospiraceae in EtOH-fed mice (Fig. 5E), while dietary B. subtilis supplementation decreased this trend. Members of the Lachnospiraceae family are important butyrate producers that reside in the intestinal microbiota (42). Furthermore, in the EtOH groups, the relative increase in Bacteroide abundance and decrease in Ruminococcaceae_UCG-013 abundance at the 

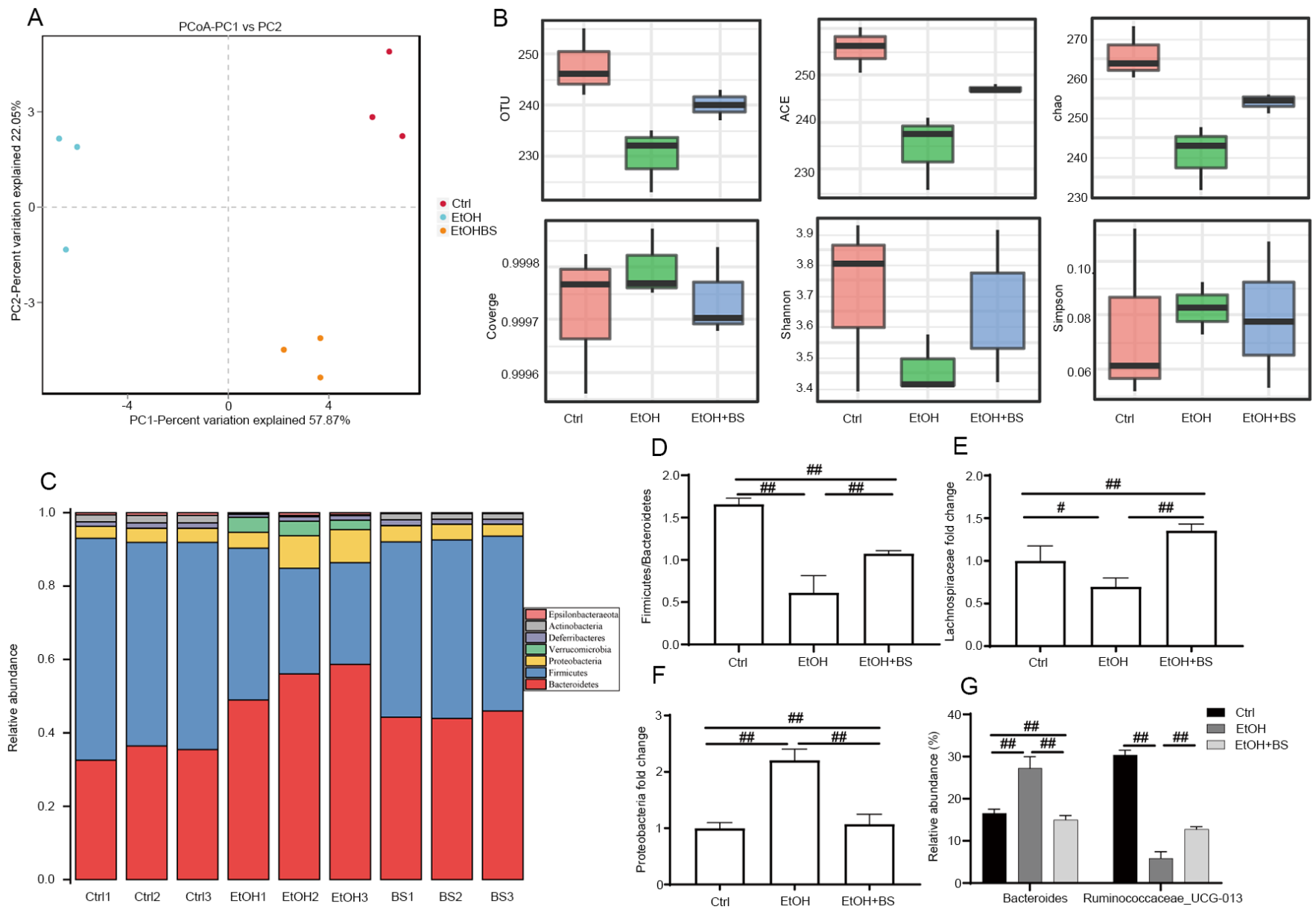

Figure 5. BS supplementation improves alcohol-induced intestinal dysbiosis. (A) Effect of BS supplementation on $\beta$ diversity (variation of microbial communities between samples) of fecal microbiota as assessed by principal coordinate analysis based on Bray-Curtis distance. (B) Effect of BS supplementation on $\alpha$ diversity (variation of microbes in a single sample) of fecal microbiota, including the indexes of OTU, ACE and chao. (C) Effect of BS supplementation on microbiota composition at the phylum level. (D) Firmicutes/Bacteroidetes ratio. (E) Lachnospiraceae (family) levels. (F) Proteobacteria (phylum) levels. (G) Bacteroides and Ruminococcaceae_UCG-013 (species) levels. Data are presented as the mean \pm standard deviation. ${ }^{\#} \mathrm{P}<0.05$; ${ }^{\# \#} \mathrm{P}<0.01$. BS, B. subtilis supplementation; Ctrl, control; EtOH, ethyl alcohol; OTU, operational taxonomic units; ACE, abundance-based coverage estimator.

species level were reversed by dietary $B$. subtilis supplementation (Fig. 5G). In summary, dietary B. subtilis supplementation partly alleviated alcohol-induced intestinal microbiota dysbiosis.

\section{Discussion}

Changes in genetic, environmental and dietary factors, as well as alcohol consumption, contribute toward intestinal dysbiosis and even severe liver diseases via the gut-liver axis $(43,44)$. Furthermore, the gut-liver axis facilitates close functional and bidirectional communication between the intestine and the liver, which serves an important role in hepatic pathogenesis and therapeutic targets $(45,46)$. Although $B$. subtilis-based probiotic supplementation may improve perturbed gut microbiota and disordered intestinal barriers, the protective effects of dietary B. subtilis supplementation on hepatic pathogenesis remain unclear.

The robust intestinal barrier serves a vital role by spatially compartmentalizing bacteria in the lumen through the secretion of mucus and is fortified by the production of SIgA and AMPs (47). Within the gut lumen, SIgA interacts with various intestinal antigens, including self-antigens and the intestinal microbiota and limits the access of intestinal antigens to blood circulation, thereby influencing the composition of intestinal microbiota $(48,49)$. Alcohol consumption notably inhibits the excretion of SIgA, thereby resulting in the overgrowth of opportunistic pathogenic enteric bacteria. Dietary B. subtilis supplementation quickly depletes free oxygen present in the intestine and produces antibacterial substances, which constrains pathogen overgrowth and restores gut microbiota homeostasis. Additionally, multiple AMPs (defensins, lysozymes and C-type lectins) are produced by epithelial cells of intestinal tissues and contribute toward the host defence against microorganisms via diverse innate immune responses (50). Among these AMPs, REG3 lectins (particularly REG3b and REG3g) are abundantly expressed in enterocytes and exert bactericidal activity as a host defence mechanism in the gut (51). The expression of REG3 lectins is inhibited by various factors, including antibiotics, alcohol and a high-fat diet $(52,53)$. By contrast, supplementation with specific probiotics enhances the expression of Reg3g, and this finding is confirmed in the present study by the partial restoration of $\operatorname{Reg} 3 b$ and $\operatorname{Reg} 3 g$ expression following dietary B. subtilis supplementation.

Efforts investigating potential therapies for alcohol-induced liver injury have been ongoing for decades, and dietary probiotic or prebiotic supplementation is a major strategy. As stated 
above, alcohol-induced liver injury is associated with gut barrier dysfunction. Furthermore, animal studies have demonstrated that dietary B. subtilis supplementation decreased the LPS translocation and abrogated the endotoxin signalling cascade, thereby attenuating alcohol-induced hepatic cytokine production, inflammatory responses and liver damage. Probiotics prevent and ameliorate the course of digestive disorders and may be of interest as co-adjuvants in the treatment of metabolic disorders, including obesity, ALD and non-alcoholic fatty liver disease $(54,55)$. However, the mechanisms of action of probiotics are diverse and strain specific. In general, supplementation with dietary probiotics normalizes perturbed intestinal microbial communities, competitively excludes enteric pathogens and bacteriocin production, and suppresses intestinal inflammation via the downregulation of TLR expression in enterocytes (56). Probiotics are safely tolerated, and several probiotics, including Lactobacillus GG, L. acidophilus, L. bulgaricus, B. bifidum, B. longum and Streptococcus thermophilus, have been demonstrated to be effective in the context of alcohol-induced liver injury (57-59). It is well recognized that probiotics supplementation may primarily ameliorate alcohol-induced intestinal dysbiosis.

Clinical and mouse model studies have revealed that alcohol causes hepatocellular damage through oxidative stress and lipids metabolism disorder $(16,60)$. Specifically, alcohol or its metabolites directly disturbs the redox homeostasis in liver, resulting in overproduction of lipid peroxidation end-products, including MDA. Therefore, the restoration of liver function is partially due to the decrease in oxidative stress (MDA) and the increases in antioxidant responses (GSH) in livers, which may be mediated by the nuclear factor erythroid 2-related factor 2 (NRF2) (61,62). Additionally, the administration of B. subtilis significantly reduces the gene expression of CD36 and PPAR- $\gamma$, which likely restores the efficient transport of triglycerides in liver. In this context, the suppression of fatty acid oxidation is likely alleviated via the transcription factor SREBP-1c and PPAR- $\alpha$, which may be able to decrease the accumulation of triglycerides in the liver and participate in preventing mitochondrial dysfunction $(63,64)$.

The results of the present study clearly demonstrated that the prophylactic intervention of $B$. subtilis partially restored the gut microbiota homeostasis and protected the intestinal tract against alcohol abuse, and the intact intestinal barrier decreased the translocation of bacterial endotoxin (LPS), which serve a crucial role in alleviating the hepatic inflammation via TLR4 signaling pathways. The present study provides the basis for future evaluations of the nutritional application of B. subtilis in the prevention and management of clinical alcohol-induced liver injury and pave the way for developing B. subtilis-based probiotics as the food additives.

\section{Acknowledgements}

Not applicable.

\section{Funding}

The present study was supported by the Affiliated Hospital of Weifang Medical University, the Weifang Medical University PhD Startup Fund (grant no. 2017 BSQD39), the National
Natural Science Foundation of China (grant no. 41907362) and the Project Funded by China Postdoctoral Science Foundation (grant no. 2019M651016).

\section{Availability of data and materials}

The datasets used and/or analyzed during the current study are available from the corresponding author on reasonable request.

\section{Authors' contributions}

PZ and XZ designed the research; PZ, MeZ, ZY, MoZ, WL, and NC performed research; PZ, YD and CC analyzed the data; PZ and $\mathrm{XZ}$ wrote the manuscript and confirmed the authenticity of all the raw data. All authors reviewed and approved the final manuscript.

\section{Ethics approval and consent to participate}

The animal experiments in the present study were approved by the Ethics and Clinical Research Committee of Nankai University (Project IRM-DWLL-2016121).

\section{Patient consent for publication}

Not applicable.

\section{Competing interests}

The authors declare that they have no competing interests.

\section{References}

1. World Health Organization (WHO): Global status report on alcohol and health 2018. WHO, Geneva, Switzerland, 2018.

2. Vassallo G, Mirijello A, Ferrulli A, Antonelli M, Landolfi R, Gasbarrini A and Addolorato G: Review article: Alcohol and gut microbiota-the possible role of gut microbiota modulation in the treatment of alcoholic liver disease. Aliment Pharmacol Ther 41: 917-927, 2015.

3. Avila MA, Dufour JF, Gerbes AL, Zoulim F, Bataller R, Burra P, Cortez-Pinto H, Gao B, Gilmore I, Mathurin P, et al: Recent advances in alcohol-related liver disease (ALD): Summary of a gut round table meeting. Gut 69: 764-780, 2020.

4. Bajaj JS: Alcohol, liver disease and the gut microbiota. Nat Rev Gastroenterol Hepatol 16: 235-246, 2019.

5. Zhong W and Zhou Z: Alterations of the gut microbiome and metabolome in alcoholic liver disease. World J Gastrointest Pathophysiol 5: 514-522, 2014.

6. Cai X, Bao L, Wang N, Ren J, Chen Q, Xu M, Li D, Mao R and Li Y: Dietary nucleotides protect against alcoholic liver injury by attenuating inflammation and regulating gut microbiota in rats. Food Funct 7: 2898-2908, 2016.

7. Chen J, Xuan YH, Luo MX, Ni XG, Ling LQ, Hu SJ, Chen JQ, Xu JY, Jiang LY, Si WZ, et al: Kaempferol alleviates acute alcoholic liver injury in mice by regulating intestinal tight junction proteins and butyrate receptors and transporters. Toxicology 429: 152338,2020

8. Zhou Z and Zhong W: Targeting the gut barrier for the treatment of alcoholic liver disease. Liver Res 1: 197-207, 2017.

9. Jiang Y, Chen B, Duan C, Sun B, Yang J and Yang S: Multigene editing in the escherichia coli genome via the CRISPR-Cas9 system. Appl Environ Microbiol 81: 2506-2514, 2015.

10. Addolorato G, Ponziani FR, Dionisi T, Mosoni C, Vassallo GA, Sestito L, Petito V, Picca A, Marzetti E, Tarli C, et al: Gut microbiota compositional and functional fingerprint in patients with alcohol use disorder and alcohol-associated liver disease. Liver Int 40: 878-888, 2020. 
11. Parlesak A, Schafer C, Schutz T, Bode JC and Bode C: Increased intestinal permeability to macromolecules and endotoxemia in patients with chronic alcohol abuse in different stages of alcohol-induced liver disease. J Hepatol 32: 742-747, 2000.

12. Hoque R, Farooq A, Ghani A, Gorelick F and Mehal WZ: Lactate reduces liver and pancreatic injury in Toll-like receptor- and inflammasome-mediated inflammation via GPR81-mediated suppression of innate immunity. Gastroenterology 146: 1763-1774, 2014.

13. Kageyama S, Nakamura K, Fujii T, Ke B, Sosa RA, Reed EF, Datta N, Zarrinpar A, Busuttil RW and Kupiec-Weglinski JW: Recombinant relaxin protects liver transplants from ischemia damage by hepatocyte glucocorticoid receptor: From bench-to-bedside. Hepatology 68: 258-273, 2018.

14. Cui K, Yan G, Xu C, Chen Y, Wang J, Zhou R, Bai L, Lian Z, Wei H, Sun R and Tian Z: Invariant NKT cells promote alcohol-induced steatohepatitis through interleukin-1 $\beta$ in mice. J Hepatol 62 : $1311-1318,2015$.

15. Wieser V, Tymoszuk P, Adolph TE, Grander C, Grabherr F, Enrich B, Pfister A, Lichtmanegger L, Gerner R, Drach M, et al: Lipocalin 2 drives neutrophilic inflammation in alcoholic liver disease. J Hepatol 64: 872-880, 2016

16. Osna NA, Donohue TM Jr and Kharbanda KK: Alcoholic liver disease: Pathogenesis and current management. Alcohol Res 38: 147-161, 2017.

17. You Y, Li WZ, Zhang S, Hu B, Li YX, Li HD, Tang HH, Li QW, Guan YY, Liu LX, et al: SNX10 mediates alcohol-induced liver injury and steatosis by regulating the activation of chaperone-mediated autophagy. J Hepatol 69: 129-141, 2018.

18. You M and Arteel GE: Effect of ethanol on lipid metabolism. J Hepatol 70: 237-248, 2019.

19. Valenzuela R and Videla LA: The importance of the long-chain polyunsaturated fatty acid n-6/n-3 ratio in development of non-alcoholic fatty liver associated with obesity. Food Funct 2: 644-648, 2011.

20. Zhang W, Sun Q, Zhong W, Sun X and Zhou Z: Hepatic peroxisome proliferator-activated receptor gamma signaling contributes to alcohol-induced hepatic steatosis and inflammation in mice. Alcohol Clin Exp Res 40: 988-999, 2016.

21. Mandrekar P and Szabo G: Signalling pathways in alcohol-induced liver inflammation. J Hepatol 50: 1258-1266, 2009.

22. Shimizu H, Masujima Y, Ushiroda C, Mizushima R, Taira S, Ohue-Kitano R and Kimura I: Dietary short-chain fatty acid intake improves the hepatic metabolic condition via FFAR3. Sci Rep 9: 16574-16574, 2019

23. Makki K, Deehan EC, Walter J and Bäckhed F: The impact of dietary fiber on gut microbiota in host health and disease. Cell Host Microbe 23: 705-715, 2018.

24. Tsai YL, Lin TL, Chang CJ, Wu TR, Lai WF, Lu CC and Lai HC: Probiotics, prebiotics and amelioration of diseases. J Biomed Sci 26 3, 2019

25. Vétizou M,Pitt JM, Daillère R, Lepage P, Waldschmitt N, Flament C, Rusakiewicz S, Routy B, Roberti MP, Duong CP, et al: Anticancer immunotherapy by CTLA-4 blockade relies on the gut microbiota. Science 350: 1079-1084, 2015.

26. Khochamit N, Siripornadulsil S, Sukon P and Siripornadulsil W: Antibacterial activity and genotypic-phenotypic characteristics of bacteriocin-producing Bacillus subtilis KKU213: Potential as a probiotic strain. Microbiol Res 170: 36-50, 2015.

27. Compaoré CS, Nielsen DS, Ouoba LII, Berner TS, Nielsen KF Sawadogo-Lingani H, Diawara B, Ouédraogo GA, Jakobsen M and Thorsen L: Co-production of surfactin and a novel bacteriocin by Bacillus subtilis subsp. subtilis $\mathrm{H} 4$ isolated from Bikalga, an African alkaline Hibiscus sabdariffa seed fermented condiment. Int J Food Microbiol 162: 297-307, 2013

28. Elshaghabee FMF, Rokana N, Gulhane RD, Sharma C and Panwar H: Bacillus as potential probiotics: Status, concerns, and future perspectives. Front Microbiol 8: 1490, 2017

29. Horosheva TV, Vodyanoy V and Sorokulova I: Efficacy of Bacillus probiotics in prevention of antibiotic-associated diarrhoea: A randomized, double-blind, placebo-controlled clinical trial. JMM Case Rep 1: 2014.

30. Hickson M: Probiotics in the prevention of antibiotic-associated diarrhoea and Clostridium difficile infection. Therap Adv Gastroenterol 4: 185-197, 2011.

31. Bertola A, Mathews S, Ki SH, Wang H and Gao B: Mouse model of chronic and binge ethanol feeding (the NIAAA model). Nat Protoc 8: 627-637, 2013.

32. Livak KJ and Schmittgen TD: Analysis of relative gene expression data using real-time quantitative PCR and the 2(-Delta Delta C(T)) method. Methods 25: 402-408, 2001.
33. Caporaso JG, Kuczynski J, Stombaugh J, Bittinger K, Bushman FD, Costello EK, Fierer N, Peña AG, Goodrich JK, Gordon JI, et al: QIIME allows analysis of high-throughput community sequencing data. Nat Methods 7: 335-336, 2010.

34. Hill TC, Walsh KA, Harris JA and Moffett BF: Using ecological diversity measures with bacterial communities. FEMS Microbiol Ecol 43: 1-11, 2003

35. Cho YE and Song BJ: Pomegranate prevents binge alcohol-induced gut leakiness and hepatic inflammation by suppressing oxidative and nitrative stress. Redox Biol 18: 266-278, 2018.

36. Shao T, Zhao C, Li F, Gu Z, Liu L, Zhang L, Wang Y, He L, Liu Y, Liu Q, et al: Intestinal HIF-1 $\alpha$ deletion exacerbates alcoholic liver disease by inducing intestinal dysbiosis and barrier dysfunction. J Hepatol 69: 886-895, 2018.

37. Antón M, Rodríguez-González A, Ballesta A, González N, Del Pozo A, de Fonseca FR, Gómez-Lus ML, Leza JC, García-Bueno B, Caso JR and Orio L: Alcohol binge disrupts the rat intestinal barrier: The partial protective role of oleoylethanolamide. Br J Pharmacol 175: 4464-4479, 2018.

38. Ambade A, Lowe P, Kodys K, Catalano D, Gyongyosi B Cho Y, Iracheta-Vellve A, Adejumo A, Saha B, Calenda C, et al: Pharmacological inhibition of CCR2/5 signaling prevents and reverses alcohol-induced liver damage, steatosis, and inflammation in mice. Hepatology 69: 1105-1121, 2019.

39. Ferrere G, Wrzosek L, Cailleux F, Turpin W, Puchois V, Spatz M, Ciocan D, Rainteau D, Humbert L, Hugot C, et al: Fecal microbiota manipulation prevents dysbiosis and alcohol-induced liver injury in mice. J Hepatol 66: 806-815, 2017.

40. Arab JP, Arrese M and Shah VH: Gut microbiota in non-alcoholic fatty liver disease and alcohol-related liver disease: Current concepts and perspectives. Hepatol Res 50: 407-418, 2020

41. Dubinkina VB, Tyakht AV, Odintsova VY, Yarygin KS Kovarsky BA, Pavlenko AV, Ischenko DS, Popenko AS, Alexeev DG, Taraskina AY, et al: Links of gut microbiota composition with alcohol dependence syndrome and alcoholic liver disease. Microbiome 5: 141, 2017.

42. Vital M, Penton CR, Wang Q, Young VB, Antonopoulos DA, Sogin ML, Morrison HG, Raffals L, Chang EB, Huffnagle GB, et al: A gene-targeted approach to investigate the intestinal butyrate-producing bacterialcommunity. Microbiome 1: 8, 2013.

43. Yan AW, Fouts DE, Brandl J, Stärkel P, Torralba M, Schott E, Tsukamoto H, Nelson KE, Brenner DA and Schnabl B: Enteric dysbiosis associated with a mouse model of alcoholic liver disease. Hepatology 53: 96-105, 2011.

44. Mutlu EA, Gillevet PM, Rangwala H, Sikaroodi M, Naqvi A, Engen PA, Kwasny M, Lau CK and Keshavarzian A: Colonic microbiome is altered in alcoholism. Am J Physiol Gastrointest Liver Physiol 302: G966-G978, 2012.

45. Wang L, Fouts DE, Stärkel P, Hartmann P, Chen P, Llorente C, DePew J, Moncera K, Ho SB, Brenner DA, et al: Intestinal REG3 lectins protect against alcoholic steatohepatitis by reducing mucosa-associated microbiota and preventing bacterial translocation. Cell Host Microbe 19: 227-239, 2016.

46. Tilg H, Cani PD and Mayer EA: Gut microbiome and liver diseases. Gut 65: 2035-2044, 2016.

47. Wells JM, Brummer RJ, Derrien M, MacDonald TT, Troost F, Cani PD, Theodorou V, Dekker J, Méheust A, de Vos WM, et al: Homeostasis of the gut barrier and potential biomarkers. Am J Physiol Gastrointest Liver Physiol 312: G171-G193, 2017.

48. Lindner C, Thomsen I, Wahl B, Ugur M, Sethi MK, Friedrichsen M, Smoczek A, Ott S, Baumann U, Suerbaum S, et al: Diversification of memory B cells drives the continuous adaptation of secretory antibodies to gut microbiota. Nat Immunol 16: 880-888, 2015.

49. Rogier EW, Frantz AL, Bruno ME, Wedlund L, Cohen DA, Stromberg AJ and Kaetzel CS: Secretory antibodies in breast milk promote long-term intestinal homeostasis by regulating the gut microbiota and host gene expression. Proc Natl Acad Sci USA 111: 3074-3079, 2014.

50. Brown GD, Willment JA and Whitehead L: C-type lectins in immunity and homeostasis. Nat Rev Immunol 18: 374-389, 2018.

51. Seo GY, Giles DA and Kronenberg M: The role of innate lymphoid cells in response to microbes at mucosal surfaces. Mucosal Immunol 13: 399-412, 2020

52. Guerville M, Leroy A, Sinquin A, Laugerette F, Michalski MC and Boudry G: Western-diet consumption induces alteration of barrier function mechanisms in the ileum that correlates with metabolic endotoxemia in rats. Am J Physiol Endocrinol Metab 313: E107-E120, 2017. 
53. van Ampting MT, Loonen LM, Schonewille AJ, Konings I, Vink C, Iovanna J, Chamaillard M, Dekker J, van der Meer R, Wells JM and Bovee-Oudenhoven IMJ: Intestinally secreted C-type lectin Reg3b attenuates salmonellosis but not listeriosis in mice. Infect Immun 80: 1115-1120, 2012.

54. Eslamparast T, Eghtesad S, Hekmatdoost A and Poustchi $\mathrm{H}$ : Probiotics and nonalcoholic fatty liver disease. Middle East J Dig Dis 5: 129-136, 2013

55. Koutnikova H, Genser B, Monteiro-Sepulveda M, Faurie MJ, Rizkalla S, Schrezenmeir J and Clément K: Impact of bacterial probiotics on obesity, diabetes and non-alcoholic fatty liver disease related variables: A systematic review and meta-analysis of randomised controlled trials. BMJ Open 9: e017995, 2019.

56. Hong M, Han DH, Hong J, Kim DJ and Suk KT: Are probiotics effective in targeting alcoholic liver diseases? Probiotics Antimicrob Proteins 11: 335-347, 2019.

57. Wang J, Dong X, Shao Y, Guo H, Pan L, Hui W, Kwok LY, Zhang H and Zhang W: Genome adaptive evolution of Lactobacillus casei under long-term antibiotic selection pressures. BMC Genomics 18: $320,2017$.

58. Koga H, Tamiya Y, Mitsuyama K, Ishibashi M, Matsumoto S, Imaoka A, Hara T, Nakano M, Ooeda K, Umezaki Y and Sata M: Probiotics promote rapid-turnover protein production by restoring gut flora in patients with alcoholic liver cirrhosis. Hepatol Int 7 : 767-774, 2013.

59. Forsyth CB, Farhadi A, Jakate SM, Tang Y, Shaikh M and Keshavarzian A: Lactobacillus GG treatment ameliorates alcohol-induced intestinal oxidative stress, gut leakiness, and liver injury in a rat model of alcoholic steatohepatitis. Alcohol 43: 163-172, 2009.

60. Gao B, Xu MJ, Bertola A, Wang H, Zhou Z and Liangpunsakul S: Animal models of alcoholic liver disease: Pathogenesis and clinical relevance. Gene Expr 17: 173-186, 2017.
61. Barrera C, Valenzuela R, Rincón M, Espinosa A, Echeverria F, Romero N, Gonzalez-Mañan D and Videla LA: Molecular mechanisms related to the hepatoprotective effects of antioxidant-rich extra virgin olive oil supplementation in rats subjected to short-term iron administration. Free Radic Biol Med 126: 313-321, 2018.

62. Valenzuela R, Illesca P, Echeverría F, Espinosa A, Rincón-Cervera MA, Ortiz M, Hernandez-Rodas MC, Valenzuela A and Videla LA: Molecular adaptations underlying the beneficial effects of hydroxytyrosol in the pathogenic alterations induced by a high-fat diet in mouse liver: PPAR- $\alpha$ and Nrf2 activation, and NF- $\mathrm{kB}$ down-regulation. Food Funct 8: 1526-1537, 2017.

63. Ortiz M, Soto-Alarcón SA, Orellana P, Espinosa A, Campos C, López-Arana S, Rincón MA, Illesca P, Valenzuela R and Videla LA: Suppression of high-fat diet-induced obesity-associated liver mitochondrial dysfunction by docosahexaenoic acid and hydroxytyrosol co-administration. Dig Liver Dis 52: 895-904, 2020.

64. Soto-Alarcón SA, Ortiz M, Orellana P,Echeverría F, Bustamante A, Espinosa A, Illesca P, Gonzalez-Mañán D, Valenzuela R and Videla LA: Docosahexaenoic acid and hydroxytyrosol co-administration fully prevents liver steatosis and related parameters in mice subjected to high-fat diet: A molecular approach. Biofactors 45: 930-943, 2019.

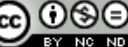

\footnotetext{
This work is licensed under a Creative Commons Attribution-NonCommercial-NoDerivatives 4.0 International (CC BY-NC-ND 4.0) License.
} 